\title{
Atopisches Ekzem geht auf die Knochen
}

\author{
Wer an einem atopischen Ekzem leidet, hat offenbar ein deutlich höheres Risiko, einen Knochenbruch \\ zu erleiden, als hautgesunde Menschen. Dabei scheint die Schwere der Hauterkrankung entscheidend für das \\ Maß der Risikosteigerung zu sein.
}

Trockene, schuppige Haut, ein ausgeprägter Juckreiz und Schmerzen, das sind die typischen Symptome eines atopischen Ekzems. Das ist offenbar nicht alles. Mit der chronisch-entzündlichen Hauterkrankung steigt das Frakturrisiko, wie eine große bevölkerungsbasierte Kohortenstudie aus Großbritannien nun ergab. Schon frühere Studien legten einen Zusammenhang zwischen atopischer Dermatitis und Knochengesundheit nahe; etwa eine Erhebung aus Taiwan aus dem Jahr 2017.

Allerdings blieb eine ganze Reihe wichtiger Fragen bislang ungeklärt, wie etwa die nach dem zeitlichen Zusammenhang, der Bedeutung der Schwere der Hauterkrankung sowie nach wichtigen Ko-Faktoren. Diese Fragen versuchte das Forscherteam um Katherine Lowe von der London School of Hygiene an Tropical Medicine nun zu klären [1].

Dazu stellten die Wissenschaftler Medizinde rund 526.000 Menschen ab 18 Jahre mit

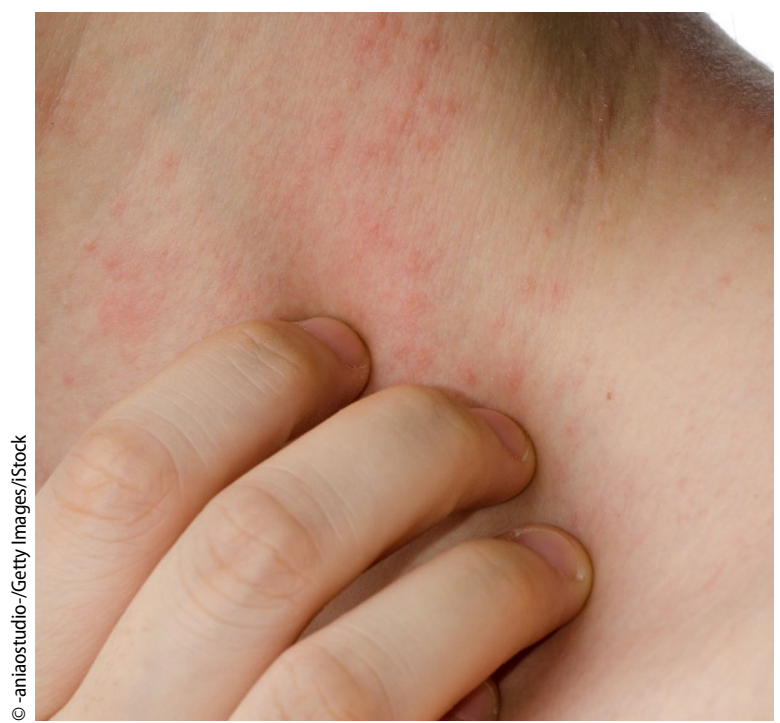

Abb. $1 \Delta$ Patienten mit schwerer Neurodermitis sollten regelmäßig ihre Knochendichte messen lassen

\section{Das Wichtigste in Kürze}

Hat das atopische Ekzem Auswirkungen auf die Knochengesundheit?

Mit der chronisch-entzündlichen Hauterkrankung steigt unabhängig von einer Glukokortikoideinnahme das Risiko für Hüft-, Becken-, Wirbel- und Knöchelfrakturen, wobei das Risiko mit der Schwere der Hauterkrankung assoziiert ist.

Bedeutung: Bei Patienten mit stark ausgeprägter atopischer Dermatitis sollte die Knochendichte gemessen werden.

Einschränkung: Einige für die Knochengesundheit wichtige Kofaktoren wie etwa die Ernährung konnten nicht berücksichtigt werden.

atopischem Ekzem 2.569.000 hautgesunden Kontrollpersonen gegenüber, die in Alter, Geschlecht und Grunderkrankungen übereinstimmten. Sie verglichen das Risiko beider Gruppen für osteoporotisch bedingte Frakturen. Neben Frakturen des Femurs, der Hüfte und des Beckens zählten die Forscher auch Wirbelfrakturen und Frakturen des Knöchels dazu. Die Daten stammten aus den Datenbanken Clinical Practice Research Datalink (CPRD Gold) und Hospital Episode Statistics (HES).

\section{Je ausgeprägter das Ekzem, desto höher das Frakturrisiko}

Probanden mit Ekzem hatten ein um $10 \%$ höheres Hüft- sowie Beckenfrakturrisiko. Das Risiko einer Wirbelkörperfraktur stieg mit der Hauterkrankung um $18 \%$, das Knöchelfrakturrisiko um $7 \%$. Kein Unterschied war bei Femurhalsfrakturen auszumachen.

Das Ausmaß der Risikosteigerung war mit dem Schweregrad der Hauterkrankung assoziiert. Am deutlichsten machte sich das bei Wirbelkörper-, Becken und Hüftfrakturen bemerkbar: Im Vergleich zu hautgesunden Probanden hatten Patienten mit ausgeprägter atopischer Dermatitis ein doppelt so hohes Wirbelkörperfrakturrisiko. Das Beckenfrakturrisiko lag $66 \%$ höher, das Hüftfrakturrisiko $50 \%$ höher. Dieser Zusammenhang blieb bei Berücksichtigung der Einnahme oraler Glukokortikoide unverändert.

\section{Knochendichte-Screening für schwer erkrankte Hautpatienten} Angesichts der weiten Verbreitung der atopischen Dermatitis und der Bedeutung osteoporotisch bedingter Frakturen auf die Lebensqualität fordern die Wissenschaftler, bei Patienten mit einer schweren Ausprägung der chronisch-entzündlichen Hauterkrankung regelmäßig die Knochendichte zu messen und dieses Patientenkollektiv in den Leitlinien zum KnochendichteScreening künftig zu berücksichtigen (• Abb. 1).

\section{Literatur}
1. Lowe KE et al (2019) Atopic eczema and fracture risk in adults: A population-based cohort study. J Allergy Clinlmmunol. https:// doi.org/10.1016/j.jaci.2019.09.015

Hinweis des Verlags. Der Verlag bleibt in Hinblick auf geografische Zuordnungen und Gebietsbezeichnungen in veröffentlichten Karten und Institutsadressen neutral.

hautnah $2020 \cdot 19: 52$

https://doi.org/10.1007/s12326020-00368-9

(c) Springer-Verlag GmbH Austria, ein Teil von Springer Nature 2020 